\section{ECG in severe hyperkalaemia: pacemaker doesn't matter so much}

Case 1: A 51-year-old man with diabetes was referred for primary angioplasty with the working diagnosis of inferior ST elevation infarction. He denied chest pain but presented with severe diarrhoea in the previous days with obnubilation. ECG showed widened QRS complexes followed by peaked Twaves with a shortened QT interval, P waves were not discernible (figure 1A). Blood analysis confirmed severe hyperkalaemia $(\mathrm{K}+9.4 \mathrm{mmol} / \mathrm{L})$ due to acute renal failure (serum creatinine $15.7 \mathrm{mg} / \mathrm{dL}$ ), without signs of ketoacidosis. Troponin I was normal. After infusion of calcium gluconate, higher heart rate and narrower QRS complexes were observed, whereas $P$ waves were sill unnoticeable (figure 1B).

Case 2: An 80-year-old woman was admitted for pacemaker lead infection treatment. Spironolactone was started due to mild heart failure. Infection was being controlled by antibiotic treatment, but 3 days later she started with progressive obnubilation. ECG showed pacemaker spikes followed by widened QRS complexes fused with tall $\mathrm{T}$ waves, similar to a sine wave pattern (figure 2A). ${ }^{1}$ Potassium was $8.4 \mathrm{mmol} / \mathrm{L}$ and renal function was normal.
Both patients received treatment for hyperkalaemia and had a progressive normalisation of consciousness and ECG pattern (figures 1C, 2B). No temporary pacemaker implantation was necessary.

As observed in our cases, the most common causes of hyperkalaemia are renal disease and medications. Less common causes are diabetic ketoacidosis, rhabdomyolysis, digoxin toxicity, large burns and tumour lysis syndrome. ${ }^{2}$

Peaked Twaves, prolongation of PR interval, absence of P wave, progressive widening of QRS, intraventricular block with bizarre morphology, pseudo Brugada pattern, elevation of ST segment, QT shortening and 'sine-wave' pattern are ECG changes welldescribed in the literature. ${ }^{3}$ These findings have an electrophysiological explanation. Potassium is a strong determinant of resting membrane potential. Increasing values of extracellular $\mathrm{K}+$ leads to a partial depolarisation of cellular membrane. This decreases the rate of rise of Phase 0 of action potential and, consequentially, causes slowing of the conduction (prolongation of PR interval and QRS widening). In addition, hyperkalaemia produces shortening of the action potential by increasing the slope of Phases 2 and 3. This corresponds to the shortening of repolarisation which can be observed in the ECG. ${ }^{4}$

There is scarce information upon whether these signs can be documented in paced rhythms. In these cases, both showed similar QRS-T wave changes secondary to severe hyperkalaemia, in spite of pacemaker stimulation.

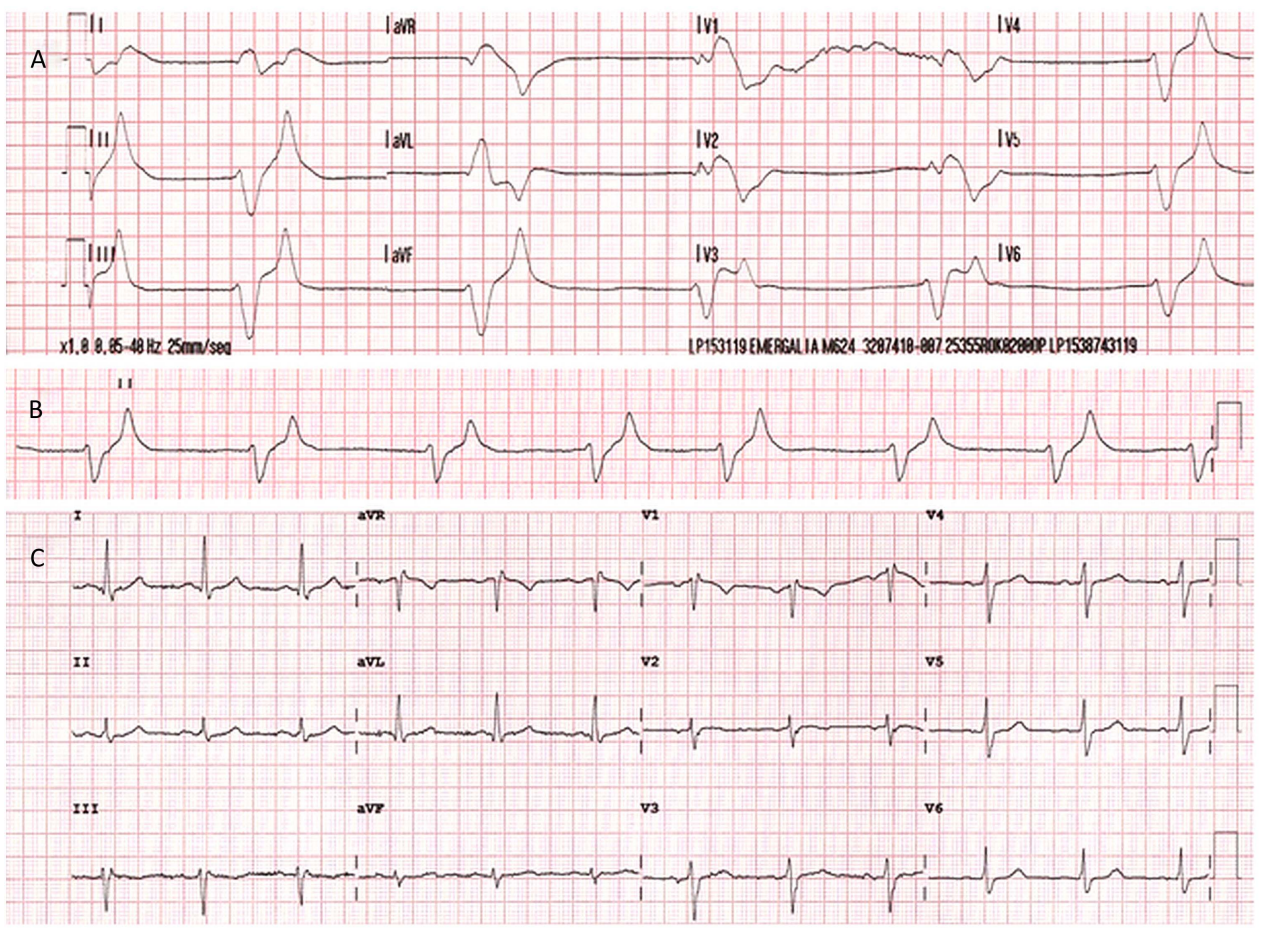

Figure 1 ECG of a spontaneous rhythm. During severe hyperkalaemia (A), after calcium gluconate administration (B) and after serum potassium normalisation (C). 


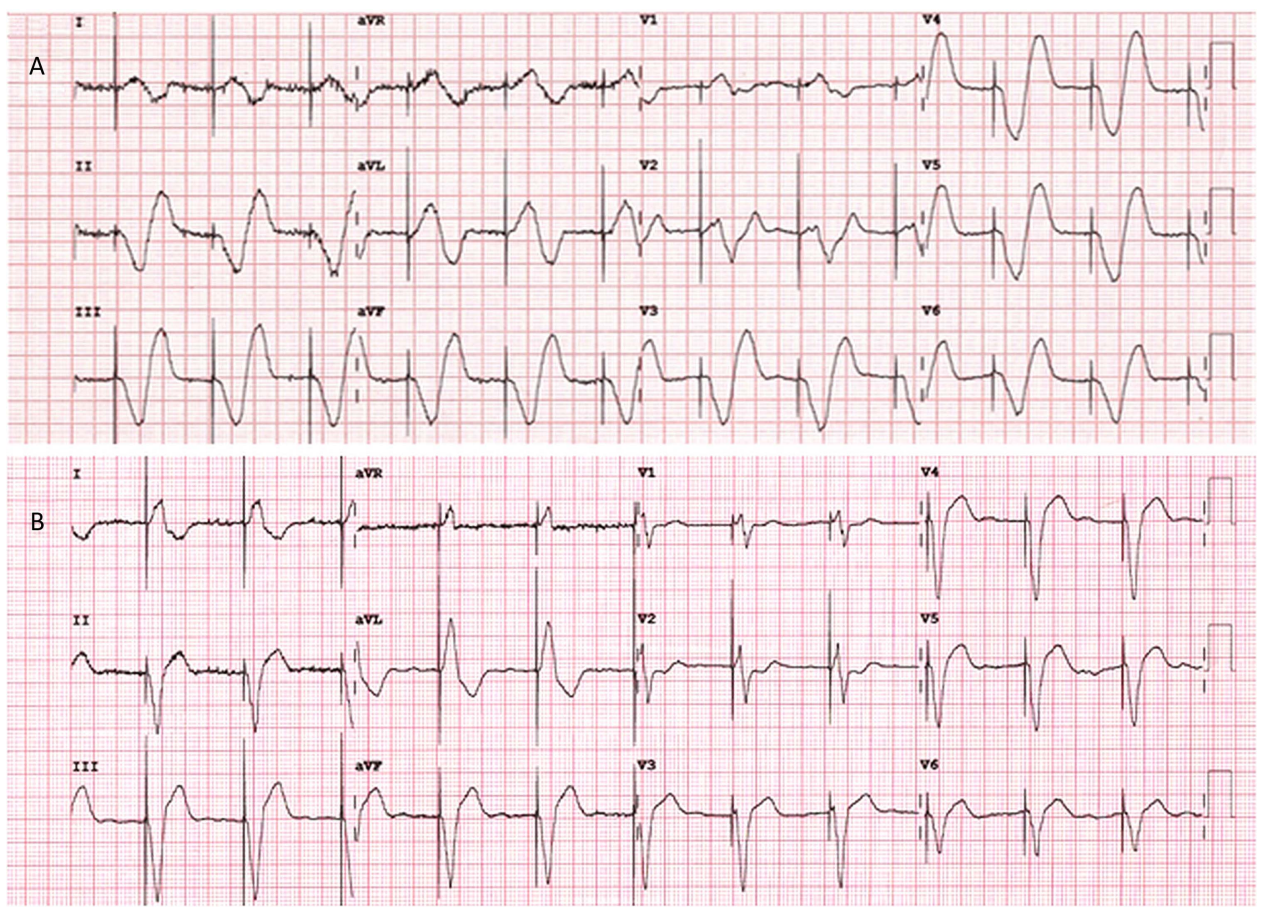

Figure 2 ECG of a paced rhythm. During severe hyperkalaemia (A) and after serum potassium normalisation (B).

\section{Alberto Cecconi, Eduardo Franco Díez, José Juan Gómez de Diego, Iván Núñez Gil}

Department of Cardiology, Cardiovascular Institute, Hospital Clínico San Carlos, Madrid, Spain

This author takes responsibility for all aspects of the reliability and freedom from bias of the data presented and their discussed interpretation.

Correspondence to Dr Alberto Cecconi, Department of Cardiology, Hospital Clínico San Carlos, C/ Profesor Martín Lagos s/n, Madrid 28040, Spain; albertocecconi@hotmail.com.

Contributors $A C$ and EFD wrote the main text, JJGdD supervised the first case report and ING supervised the second case report.

Competing interests None.

Patient consent Obtained.

Provenance and peer review Not commissioned; externally peer reviewed.

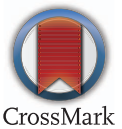

To cite Cecconi A, Franco Díez E, Gómez de Diego JJ, et al. Heart Asia 2014;6:46-47. doi:10.1136/heartasia-2013-010384

Heart Asia 2014;6:46-47. doi:10.1136/heartasia-2013-010384

\section{REFERENCES}

1 Petrov DB. An electrocardiographic sine wave in hyperkalemia. N Engl J Med 2012;366:1824.

2 Acker CG, Johnson JP, Palevsky PM, et al. Hyperkalemia in hospitalized patients: causes, adequacy of treatment, and results of an attempt to improve physician compliance with published therapy guidelines. Arch Intern Med 1998;158:917-24.

3 Mattu A, Brady WJ, Robinson DA. Electrocardiographic manifestations of hyperkalemia. Am J Emerg Med 2000;18:721-9.

4 Barold SS, Herweg B. The effect of hyperkalaemia on cardiac rhythm devices. Europace 2014. [Epub ahead of print]. 